\title{
Quasi-phase-matched acceleration of electrons in a corrugated plasma channel
}

\author{
S. J. Yoon, ${ }^{1}$ J. P. Palastro, ${ }^{1}$ D. Gordon, ${ }^{2}$ T. M. Antonsen, ${ }^{1}$ and H. M. Milchberg ${ }^{1}$ \\ ${ }^{1}$ Institute for Research in Electronics and Applied Physics, University of Maryland, College Park, Maryland 20740, USA \\ ${ }^{2}$ Naval Research Laboratory, Washington, D.C. 20375, USA
}

(Received 25 March 2012; published 9 August 2012)

\begin{abstract}
A laser pulse propagating in a corrugated plasma channel is composed of spatial harmonics whose phase velocities can be subluminal. The phase velocity of a spatial harmonic can be matched to the speed of a relativistic electron resulting in direct acceleration by the guided laser field in a plasma waveguide and linear energy gain over the interaction length. Here we examine the fully self-consistent interaction of the laser pulse and electron beam using particle-in-cell (PIC) simulations. For low electron beam densities, we find that the ponderomotive force of the laser pulse pushes plasma channel electrons towards the propagation axis, which deflects the beam electrons. When the beam density is high, the space charge force of the beam drives the channel electrons off axis, providing collimation of the beam. In addition, we consider a ramped density profile for lowering the threshold energy for trapping in a subluminal spatial harmonic. By using a density ramp, the trapping energy for a normalized vector potential of $a_{0}=0.1$ is reduced from a relativistic factor $\gamma_{0}=170$ to $\gamma_{0}=20$.
\end{abstract}

DOI: 10.1103/PhysRevSTAB.15.081305

PACS numbers: 52.38.Kd, 41.75.Ht, 52.40.Fd, 41.75.Jv

\section{INTRODUCTION}

In this paper, we explore, through theory and simulations, the quasi-phase-matched direct acceleration (QPMA) of electrons in an axially modulated plasma waveguide. Quasi-phase-matching refers to matching the electron velocity to the phase velocity of an individual spatial harmonic comprising an electromagnetic wave propagating in an axially modulated structure. In analogy with quasi-phasematching in nonlinear optics, we use a periodic medium to compensate the difference between the electron velocity and the phase velocity of the accelerating field to transfer energy from the accelerating field to the electron. In nonlinear optics, perfect phase-matching requires the phase velocity of the nonlinear source term (the nonlinear polarization) to be equal to that of the generated light, such as the second harmonic; equivalently, the wave vectors of these two waves are equal. In the absence of phase-matching, a modulation in the nonlinearity or in the refractive index can be introduced along the propagation axis (axial modulation) such that the wave vector mismatch (phase velocity mismatch) between the two waves is provided by the effective wave vector of the structure. This allows net energy transfer from the nonlinear polarization wave to the desired product electromagnetic wave [1]. For direct laser acceleration of electrons, perfect phase-matching would require matching the electron velocity to the phase velocity of the accelerating electromagnetic wave. However, because an electron cannot gain energy from an electromagnetic wave in an axially

Published by the American Physical Society under the terms of the Creative Commons Attribution 3.0 License. Further distribution of this work must maintain attribution to the author(s) and the published article's title, journal citation, and DOI. uniform propagation medium or structure, one must introduce an axially periodic structure that compensates the velocity mismatch. The principle of matching the period of the structure to the dephasing length is required in both the nonlinear optics case and the electromagnetic wave + electron beam case.

In the scheme described here, an electron beam copropagating with a radially polarized laser pulse injected into a corrugated plasma waveguide is accelerated by a phasematched axial spatial harmonic of the resulting guided electromagnetic field, providing linear energy gain over the interaction length [2]. In order to achieve linear energy gain over an extended interaction length, three things are required: slow electromagnetic waves (providing quasiphase-matching), a channel for guiding the laser pulse (eliminating diffractive spreading of the laser pulse) $[3,4]$, and radial polarization (providing a component of electric field along the propagation axis) [2].

In uniform plasma, the phase velocity of a light wave is greater than the speed of light in vacuum making linear, direct acceleration of charged particles with laser pulses impossible. This is an extension of the Lawson-Woodward theorem [5], which states that the energy gain of an electron accelerated by the linear field of a laser pulse in vacuum over an infinite distance is zero. Linear acceleration can however be achieved through quasi-phasematching of electromagnetic waves to charged particles. Quasi-phase-matching requires a structure whose dispersion allows for "slow waves" or components of the electromagnetic wave with subluminal phase velocities. Layer et al. succeeded in imparting axial density modulations on a miniature plasma channel creating an ideal structure for quasi-phase-matching [6,7]. The longitudinal periodicity of the structure provides dispersion where a single 
frequency is associated with a spectrum of axial wave numbers, some associated with subluminal phase velocities. In addition to providing slow wave dispersion, the corrugated plasma channel provides a guiding structure for laser pulses. As a plasma, it eliminates the damage limitation typical of metallic guides or solid-state dielectric guiding structures. Even with slow wave dispersion, a linearly polarized laser pulse cannot accelerate electrons along the axis of the channel without an appropriate field component in the acceleration direction. The axial field associated with a linearly polarized laser pulse is zero on axis. A radially polarized laser pulse, on the other hand, has a longitudinal component whose transverse dependence has a maximum on axis. This longitudinal component is obtained by Gauss' law, giving $(1 / r) \partial\left(r E_{r}\right) / \partial r=-\partial E_{z} / \partial z$ for cylindrical symmetry.

To illustrate the principle of QPMA and find a scaling for energy gain, we consider a simple model presented by Palastro et al. [8,9]. The radial component of the laser pulse vector potential in the plasma channel can be expressed in envelope form as follows:

$$
A_{\perp}=\hat{A}_{r}(r, z, t) \exp \left[i\left(k_{0} z-\omega_{0} t\right)\right]+\text { c.c., }
$$

where $k_{0}$ and $\omega_{0}=c k_{0}$ are the central wave number and frequency of the laser pulse, respectively, and $\hat{A}_{r}$ is the envelope function. Azimuthal symmetry of the laser pulse is assumed. We consider channel electron densities with $\omega_{p} \ll \omega_{0}$, where $\omega_{p}^{2}=4 \pi e^{2} n_{e}(r, z) / m_{e}$ is the plasma frequency squared, and $m_{e}$ and $e$ are the electron mass and the electron charge. In this limit, the temporal and spatial variation of $\hat{A}_{r}$ during propagation are slow compared to $\omega_{0}$ and $k_{0}$, respectively, and the slowly varying envelope equation can be used to describe the evolution of the laser pulse:

$$
\left[2 i k_{0}\left(\frac{\partial}{\partial z}+\frac{1}{c} \frac{\partial}{\partial t}\right)+\frac{1}{r} \frac{\partial}{\partial r} r \frac{\partial}{\partial r}-\frac{1}{r^{2}}\right] \hat{A}_{r}=\frac{\omega_{p}^{2}(r, z)}{c^{2}} \hat{A}_{r} .
$$

Equation (2) includes only the linear plasma response to the vector potential, thus our model is limited to the consideration of nonrelativistic vector potentials $a_{0}=$ $e A_{0} / m_{e} c^{2}<1$, where $A_{0}$ is the amplitude of $A_{\perp}$. We consider the following density profile mimicking the experimental results of Layer et al. [6,7]:

$$
n_{e}(r, z)=n_{0}\left[1+\Gamma \sin \left(k_{m} z\right)\right]+\frac{1}{2} n_{0}^{\prime \prime} r^{2},
$$

where $\Gamma$ is modulation amplitude and $n_{0}^{\prime \prime}$ determines the curvature of the channel.

The lowest order transverse mode solution of Eq. (2) is

$$
\begin{aligned}
\hat{A}_{r}(r, z, t)= & A_{0} \frac{r}{w_{\mathrm{ch}}} e^{-r^{2} / w_{\mathrm{ch}}^{2}-\left(z-v_{g} t\right)^{2} / \sigma_{z}^{2}} \\
& \times \sum_{n} i^{n} J_{n}(\psi) e^{-i \psi+i\left(\delta k+n k_{m}\right) z},
\end{aligned}
$$

where $\sigma_{z}$ is the axial extent of the laser pulse, $w_{\mathrm{ch}}^{2}=$ $2 c\left(2 / \omega_{p 0}^{\prime \prime 2}\right)^{1 / 2}$, where $\omega_{p 0}^{\prime \prime 2}=\left(e^{2} / m_{e}\right) n_{0}^{\prime \prime}, J_{n}(\psi)$ is the $n$th
Bessel function of the first kind, $\psi=\Gamma \omega_{p 0}^{2} / 2 c^{2} k_{0} k_{m}$, and $\delta k=-k_{0}^{-1}\left(\omega_{p 0}^{2} / 2 c^{2}+4 / w_{\mathrm{ch}}^{2}\right)$. The pulse envelope is a sum of spatial harmonics with $J_{n}(\psi)$ determining the relative amplitude. For typical experimental parameters, $\psi \ll 1$ and $J_{n}(\psi) \sim \psi^{n} / 2^{n} n$ !: the relative amplitude drops rapidly with increasing harmonic number $n$. For maximum acceleration we want to consider the $n=1$ spatial harmonic. With Eq. (4) we can extract the longitudinal accelerating field of the first spatial harmonic, using $\boldsymbol{\nabla} \cdot \mathbf{A} \simeq 0$ for $\left(\omega_{p} / \omega_{0}\right)^{2} \ll 1$ for which we find

$$
\mathbf{A}_{z, 1}(r, z, t)=i \frac{2 J_{1}(\psi)}{k_{0} w_{\mathrm{ch}}} A_{0} e^{-r^{2} / w_{\mathrm{ch}}^{2}-\left(z-v_{g} t\right)^{2} / \sigma_{z}^{2}-i \psi+i\left(\delta k+k_{m}\right) z}
$$

The corresponding phase velocity for the $n=1$ spatial harmonic is then

$$
\frac{\mathrm{v}_{p, 1}}{c} \simeq 1-\frac{k_{m}}{k_{0}}+\frac{\omega_{p 0}^{2}}{2 \omega_{0}^{2}}+\frac{4}{\left(k_{0} w_{\mathrm{ch}}\right)^{2}},
$$

where we have assumed $\left|k_{0}\right| \gg\left|k_{m}\right|,|\delta k|$. By an appropriate choice of $k_{m}$, we can tune the phase velocity for QPMA. In particular, simple analysis shows that the ideal phase velocity for a relativistic electron beam is $\mathrm{v}_{p, 1}=c$, or $k_{m}=-\delta k[10]$.

With the analytic expression for the vector potential in a corrugated waveguide, a scaling law for energy gain of an electron can be derived. A test electron with initial conditions $\left(r, \mathrm{v}_{r}, z, \mathrm{v}_{z}\right)=\left(0,0, z_{0}, \mathrm{v}_{z, 0}\right)$ is considered for the scaling law. The initial velocity of the electron, $\mathrm{v}_{z, 0}$, is taken close to $c$ so that phase-matching between the electron and the spatial harmonic is ensured over the interaction distance. Using $\Delta \gamma=-q / m_{e} c^{3} \int \mathbf{v} \cdot \partial_{t} \mathbf{A} d t$, we obtain the energy gain for a test particle in a constant laser field:

$$
\Delta \gamma \sim 2 \Gamma a_{0}\left(1+\frac{2 \lambda_{p}^{2}}{\pi^{2} w_{\mathrm{ch}}^{2}}\right)^{-1}\left(\frac{z_{\mathrm{min}}}{w_{\mathrm{ch}}}\right),
$$

where $z_{\min }$ is the minimum interaction length. In particular, the interaction length can be limited by the length of the channel, the time it takes for the electrons to travel through the laser pulse, or defocusing effects in the beam. The energy gain increases linearly with modulation amplitude, $\Gamma$, and laser amplitude, $a_{0}$, and is inversely proportional to $w_{\mathrm{ch}}$, a result of larger axial fields in narrower channels.

Recently, there has been much research activity in the area of laser plasma based acceleration of electrons. One of the most widely studied schemes is laser wakefield acceleration (LWFA) of electrons [11-19]. In LWFA, the nonlinear ponderomotive force of a high intensity laser pulse drives a strong longitudinal electrostatic wave, exceeding $10 \mathrm{GV} / \mathrm{m}$ in the plasma [16]. GeV-scale acceleration with a quasimonoenergetic peak and low emittance has been already achieved with LWFA over several centimeters [16]. In addition, many studies have shown stable control 
of output energy and charge in the accelerated bunches [17-19]. Despite a number of advantages with LWFA, it depends on a nonlinear laser plasma interaction, necessitating multi-TW laser systems. Several direct laser acceleration schemes, such as the inverse Cherenkov accelerator [20], the semi-infinite vacuum accelerator [21], and vacuum beat wave accelerator [22-24], are proposed as alternatives for small-scale laser systems. However, these schemes suffer a low acceleration gradient $(<40 \mathrm{MV} / \mathrm{m})$ and short interaction distance (vacuum diffraction length). To our knowledge, QPMA provides the highest acceleration gradients at nonrelativistic laser intensity. For our typical laboratory parameters [6,7], a QPMA acceleration gradient of $10.6 \mathrm{MV} / \mathrm{cm}$ can be achieved with a $30 \mathrm{GW}$ laser pulse. QPMA is especially attractive for modest energy, high repetition rate laser systems. Moreover, the energy gain in QPMA grows linearly with the length of plasma waveguide as long as the electrons do not outrun the laser pulse.

The goal of this paper is to study QPMA using fully selfconsistent particle-in-cell (PIC) simulations. The simulations are used to validate the simple model described above, examine the self-consistent electrostatic fields generated by the beam itself, and to conduct preliminary studies on density ramping for lowering the required injection energies in QPMA. The paper is organized as follows. In Sec. II, we describe the 2D cylindrical PIC simulation, TURBOWAVE, and validate the code for QPMA simulations. In Sec. III, we present simulation results of electron acceleration in corrugated waveguides and comparisons to analytic predictions. Section IV includes an analytic model and simulations of reduced energy threshold electron acceleration using a plasma density ramp. Section V is the conclusions.

\section{CODE DETAIL AND VERIFICATION}

In order to account for nonlinear effects, and to more fully describe the dynamics of the accelerated electrons, 2D axisymmetric particle-in-cell (PIC) simulations are used. In this work we used the code TURBOWAVE [25]. TURBOWAVE is a framework for solving the MaxwellLorentz system of equations for charged particle dynamics. The model is fully relativistic and fully electromagnetic. The fields are advanced using a straightforward extension of the usual Yee solver [26] to the case of cylindrical coordinates. The sources are deposited using linear weighting, with charge conservation ensured by means of a Poisson solver [27]. Since dynamics on the time scale of the laser period are important, the full relativistic equations of motion are solved.

The simulations are done in a moving frame, which moves at $c$. In the moving frame the coordinates are $\xi=$ $z-c t$ and $t$, where $\xi$ represents distance in the moving frame. The extent of the simulation window is $80 \mu \mathrm{m}$ in the transverse direction and $100 \mu \mathrm{m}$ in $\xi$. The number of grid points used in the transverse direction and the $\xi$ direction are 512 and 4096, respectively. The radially polarized laser pulse is initialized as a lowest order associated Laguerre Gaussian mode with a wavelength of $800 \mathrm{~nm}$, longitudinal width of $100 \mathrm{fs}$ in $e^{-1}$, and transverse radius of $15 \mu \mathrm{m}$ in $e^{-1}$. The field amplitude is varied. The pulse begins outside the channel and unless otherwise stated the plasma density is ramped up linearly over $20 \mu \mathrm{m}$. After the initial ramp, the electron density is given by the equation

$$
n_{e}(r, z)= \begin{cases}n_{0}\left[1+\Gamma \sin \left(k_{m} z\right)\right]\left(1+\frac{1}{2} n_{0}^{\prime \prime} r^{2}\right) & r<r_{c} \\ 0 & r \geq r_{c},\end{cases}
$$

where $r_{c}=70 \mu \mathrm{m}$. The channel has a parabolic density profile in the transverse direction to which the laser pulse is matched and a sinusoidal density oscillation in the longitudinal direction. The channel terminates at some radius allowing for side loss of electromagnetic energy. Four particles are loaded at each cell to represent electrons consisting a plasma waveguide. The parameters for the density profile are an average density $n_{0}=$ $7 \times 10^{18} \mathrm{~cm}^{-3}$, a modulation amplitude of $\Gamma=0.9$, and a modulation period of $356 \mu \mathrm{m}$. This choice of parameters sets the phase velocity of the 1 st spatial harmonic to $c$ based on the simple theory and are experimentally realizable [10]. Later we will show that electron energy gain is sensitive to the choice of modulation period. The ions evolve on a time scale much longer than the pulse duration and are set to be immobile in the simulation.

We examine the validity of the simple theory and 2D cylindrical PIC code by confirming the presence of spatial harmonics of a laser pulse guided in a corrugated plasma channel. This is done using two methods. The first is a Fourier decomposition of the electromagnetic field. For the Fourier decomposition we consider a small vector potential $a_{0}=0.01$ to ensure linear propagation and a deliberately small density modulation period of $2 \pi / k_{m}=20 \mu \mathrm{m}$. While this is not the optimal period for direct acceleration it is more computationally efficient for examining the spectral structure of the electromagnetic field. In particular, the wave number resolution in a discrete Fourier transform is given by $2 \pi / L$, where $L$ is the length of the moving frame box. If we wish to resolve spectral features due to the modulations, the Nyquist condition must be satisfied: $k_{m} L \gg 2 \pi$. In addition, we are required to resolve the central laser wave number: $k_{0} \Delta \xi \ll 2 \pi$. Combining these two conditions we see that $N_{\xi} \gg k_{0} / k_{m}$, where $N_{\xi}$ is the number of numerical grid points in $\xi$ : the larger the modulation period, the more computationally intensive the calculation.

For examining the spectral properties, we Fourier transform the on-axis longitudinal electric field, $E_{z}$, from the simulation in both time and space. Figure 1(a) shows the magnitude of the Fourier transform on a log scale as a 

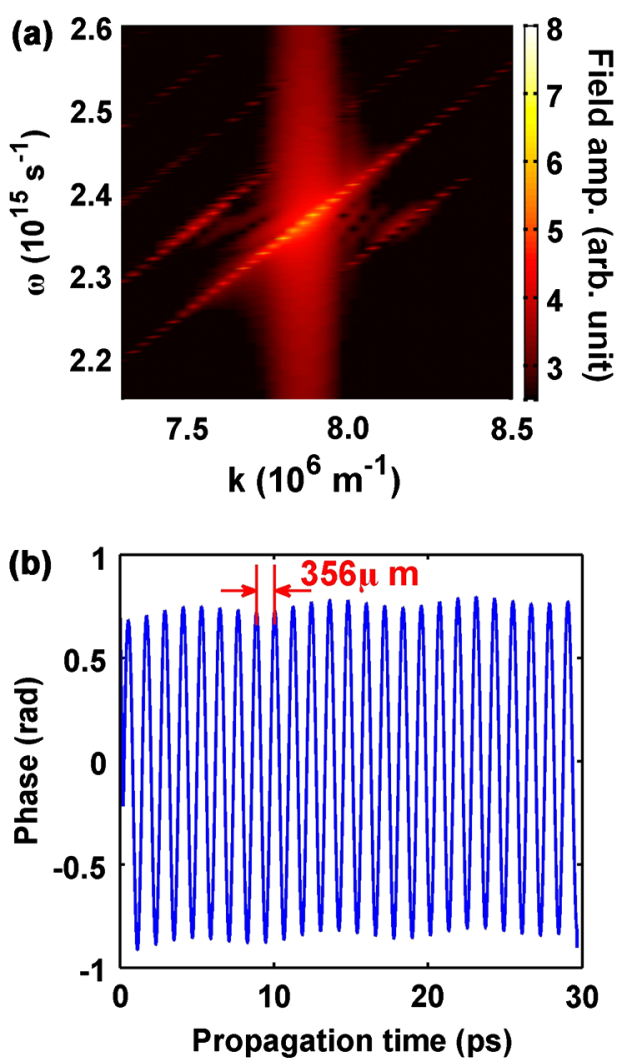

FIG. 1. (a) Fourier transform of the longitudinal electric field $\left(E_{z}\right)$ in time $(t)$ and space $(z)$ at a laser wavelength $\lambda_{L}=$ $800 \mathrm{~nm}$, normalized vector potential $a_{0}=0.01$, modulation period $d=20 \mu \mathrm{m}$, and modulation amplitude $\Gamma=0.9$. The diagonal lines represent the spatial harmonics. The fundamental spatial harmonic is the central diagonal line. The diagonals to the left and right of the fundamental are \pm 1 st spatial harmonics, respectively. (b) Phase of the on-axis longitudinal electric field at a fixed $\xi$ point. The period of the phase oscillation corresponds to the density modulation period.

function of $\omega$ and $k$. The fundamental spatial harmonic is the brightest diagonal line in Fig. 1(a) surrounded by the $n= \pm 1$ spatial harmonics. The higher order spatial harmonics, $|n|>1$, are dim in this figure. As expected the amplitude of these modes drops rapidly as $n$ increases. To compare to the simulation, we consider the theoretical ratio of the fundamental and first spatial harmonics $J_{1}(\psi) / J_{0}(\psi)$, and the simulated ratio. We consider two different density modulation amplitudes: $\Gamma=0.9$ and $\Gamma=0.2$. Table I shows excellent agreement between the simulation results and the analytical calculation. It also

TABLE I. Comparison between the ratios of the fundamental and first spatial harmonics from theory and simulation.

\begin{tabular}{lcc}
\hline \hline $\begin{array}{l}\text { Modulation } \\
\text { amplitude }\end{array}$ & $\begin{array}{c}\text { Amplitude } \\
\text { ratio (theory) }\end{array}$ & $\begin{array}{c}\text { Amplitude ratio } \\
\text { (simulation) }\end{array}$ \\
\hline$\Gamma=0.2$ & 0.0055 & 0.0051 \\
$\Gamma=0.9$ & 0.025 & 0.023 \\
\hline \hline
\end{tabular}

tells us that the modulation of plasma density in a plasma waveguide excites spatial harmonics, and the ratio is linearly proportional to the modulation amplitude.

The second method for confirming the presence of spatial harmonics is extraction of the electromagnetic field's phase. The phase contains information about the linear response of the media and more specifically the spatial profile of the background electron density. The connection between the first and second approach is manifest in the relationship: $\sum_{n} i^{n} J_{n}(\psi) \exp \left[i n k_{m} z\right]=\exp \left[i \psi \cos \left(k_{m} z\right)\right]$. Periodic phase oscillations lead to the presence of spatial harmonics. From Eq. (4) we can write the total phase of the laser pulse as $\phi_{T}(\xi, t)=\left(k_{0}+\delta k\right) \xi+c \delta k t-$ $\psi \cos \left[k_{m}(\xi+c t)\right]$. For extracting the phase from the simulation we consider a small vector potential $a_{0}=0.1$ to ensure linear propagation and a standard modulation period of $356 \mu \mathrm{m}$. A benefit of phase extraction is that there are no additional numerical requirements as with the Fourier decomposition. Again we consider the on-axis axial field. The phase is defined by

$$
\phi=\arctan \left[\frac{\operatorname{Im}\left\langle e^{i k_{0} \xi} E_{z}\right\rangle_{t}}{\operatorname{Re}\left\langle e^{i k_{0} \xi} E_{z}\right\rangle_{t}}\right],
$$

where the brackets represent an average over one period of the laser pulse and $\phi \simeq \phi_{T}-k_{0} \xi$. The averaging removes the variation at the carrier wave number leaving only the slowly evolving component. This method is only valid when the variation at $k_{0}$ is much faster than any other variation in the laser pulse: $k_{0} \gg|\delta k|, k_{m}$. Figure 1(b) shows the phase oscillation due to the channel density modulation. As expected, the oscillation period in time corresponds to the modulation period.

\section{2D PIC SIMULATION RESULTS}

In this section, we present results of fully self-consistent 2D cylindrical PIC simulations of QPMA. For QPMA the copropagating electron beam is initialized as a Gaussian in both the transverse and longitudinal directions with a transverse and longitudinal $e^{-1}$ length of 3 and $5 \mu \mathrm{m}$, respectively. Eight particles per cell are used to represent the copropagating electron beam. The beam electrons start with identical longitudinal momentum and no transverse momentum. The beam density is varied. Because of the subluminal group velocity of the laser pulse, the laser pulse will move backwards with respect to the electron beam. To maximize the electron acceleration during $1 \mathrm{~cm}$ of propagation, the beam is initially located $10 \mu \mathrm{m}$ behind the peak of the laser pulse.

We first consider the longitudinal field experienced by an on-axis, highly relativistic test electrons in a laser pulse with $a_{0}=0.1$ over $9 \mathrm{~mm}$. Figure 2(a) shows the on-axis longitudinal electric field for a fixed value of $\xi$ in blue. In a plasma channel without modulations, the oscillation would be sinusoidal with a period of $L_{d}=2 \pi /|\delta k|$, which we refer to as the dephasing length. The dephasing length is 

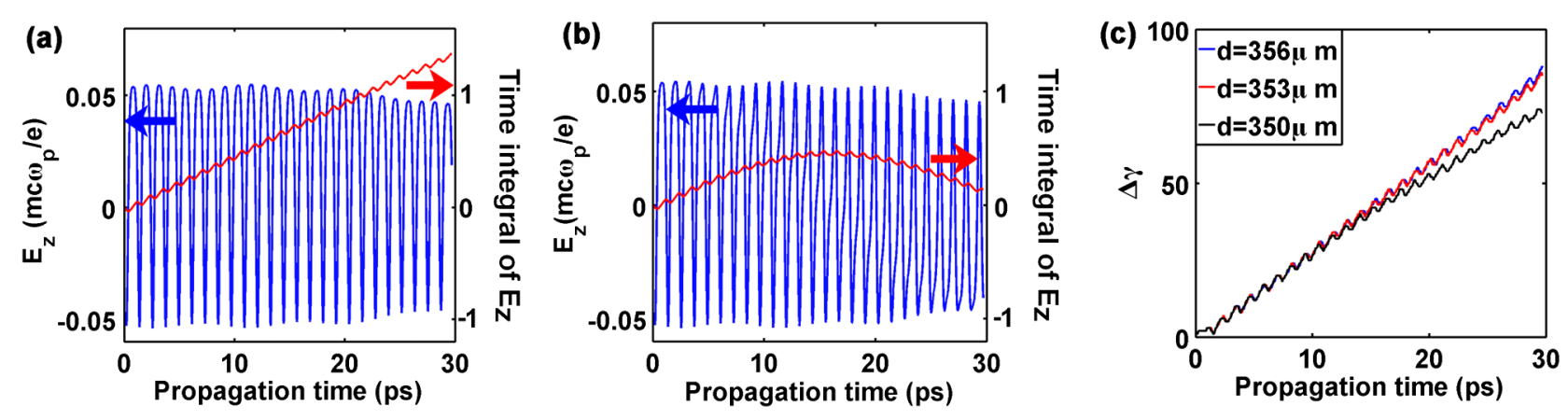

FIG. 2. On-axis longitudinal electric field (blue) and time integral of the on-axis longitudinal electric field (red) as a function of acceleration time for a fixed $\xi$ point. In (a) the modulation period matches the dephasing length, $L_{d}=356 \mu \mathrm{m}$, while in (b) the modulation period is $d=350 \mu \mathrm{m}$. (c) Self-consistent energy gain as a function of acceleration for three different modulation periods: $350 \mu \mathrm{m}, 353 \mu \mathrm{m}$, and $356 \mu \mathrm{m}$.

the distance over which an electron moving at velocity $c$ would see the laser pulse slip by one wavelength in an unmodulated channel. The oscillation here shows the phase evolution of all of the spatial harmonics except $n=1$ whose phase velocity is identically $c$. In general, each spatial harmonic will undergo a $2 \pi$ phase oscillation in a distance $L_{d}(n)=2 \pi k_{m}^{-1}(n-1)^{-1}$ for $n \neq 1$, where we have used $k_{m}=-\delta k$. The predominant mode in Fig. 2(a) is the fundamental spatial harmonic, but the presence of the other spatial harmonics is noticeable in the nonsinusoidal shape of the oscillation. The red curve in Fig. 2(a) shows the energy gain calculated from $W(\xi, t)=$ $-c e \int_{0}^{t} E_{z}(\xi, t) d t$. By setting the modulation period equal to the dephasing length, we have ensured that the $n=1$ spatial harmonic is stationary in the frame of an electron moving with a velocity near $c$. The relative phase of the electron and spatial harmonic does not change and the electron undergoes linear energy gain. The oscillations in energy are due to the electron quiver motion from the other spatial harmonics.

In Fig. 2(b) the modulation period is chosen to be smaller such that $k_{m} \neq-\delta k$ and the $n=1$ spatial harmonic no longer has a phase velocity identical to $c$. In particular, the modulation period is set to $350 \mu \mathrm{m}, 6 \mu \mathrm{m}$ less than $L_{d}$. The waveform changes noticeably over $1 \mathrm{~cm}$ propagation. Each spatial harmonic now undergoes a $2 \pi$ phase oscillation in a distance $L_{d}(n)=2 \pi\left|n k_{m}+\delta k\right|^{-1}$, which cannot be related by integer multiples for each $n$. This results in the spatial harmonics dephasing from one another and causing the waveform to change shape. Again, the red line shows the energy gain, and as expected the electron initially gains energy then loses energy as none of the spatial harmonics are stationary in the electron frame. This demonstrates that QPMA is sensitive to the matching between modulation period and the dephasing length. We note that the length of the plasma waveguide can be reduced to account for this sensitivity. For example, if the modulation period is $350 \mu \mathrm{m}$ and the plasma waveguide is $0.45 \mathrm{~cm}(12 \mathrm{ps})$ electrons will gain energy over the entire interaction length. Figure 2(c) shows self-consistent energy gains over $9 \mathrm{~mm}$ for an initial energy $\gamma_{0}=200$ and laser pulse amplitude $a_{0}=0.1$ for different modulation periods $d=350 \mu \mathrm{m}, 353 \mu \mathrm{m}$, and $356 \mu \mathrm{m}$ in black, red, and blue, respectively. The energy gain for $d=356 \mu \mathrm{m}$ and $d=353 \mu \mathrm{m}$ is almost identical. Even with $d=$ $350 \mu \mathrm{m}$ the electron gains more energy than is predicted in Fig. 2(b). The discrepancy between Fig. 2(b) and Fig. 2(c) can be explained as follows. In Fig. 2(b) the electron is assumed to move at the speed of light while the phase velocity of the $n=1$ spatial harmonic is slightly subluminal resulting in the dephasing described above. In Fig. 2(c), however, the electron is moving slightly below $c$ and remains phased with the spatial harmonic over a longer distance.

Figures 3(a) and 3(b) show simulated energy gains over $9 \mathrm{~mm}$ for different initial electron energies at pulse amplitudes of $a_{0}=0.1$ and $a_{0}=0.25$, respectively. The energy gains displayed are those of electrons having the largest energy gain of all the electrons. The energy spectra will be examined below. The red line in both figures is the result for an initial energy of $\gamma_{0}=100$. Figure 3(a) demonstrates energy gain for $\gamma_{0}=100$, but not linear energy gain whereas Fig. 3(b) shows linear energy gain over the entire $9 \mathrm{~mm}$. This is a result of the threshold energy for trapping in a spatial harmonic,

$$
\gamma_{\mathrm{th}} \simeq\left(\frac{k_{m} w_{\mathrm{ch}}}{4 \Gamma a_{0}}\right)\left(\frac{\omega}{\omega_{p, 0}}\right)^{2} .
$$

The threshold energy is the minimum energy for electrons to gain energy linearly from the laser field. For $a_{0}=0.1$ and $a_{0}=0.25$ the thresholds are $\gamma_{\mathrm{th}}=183$ and $\gamma_{\mathrm{th}}=73$, respectively: $\gamma_{0}=100$ is below the threshold energy for $a_{0}=0.1$, but above for $a_{0}=0.25$. Because it is below threshold the energy gain for $\gamma_{0}=50$ saturates for both $a_{0}=0.1$ and $a_{0}=0.25$. The energy gain of the electrons with initial energy above $\gamma_{\mathrm{th}}$ matches the prediction of the scaling law given in Eq. (7). For $a_{0}=0.25$ and an interaction length of $9 \mathrm{~mm}$, the scaling law predicts $\Delta \gamma \simeq 204$, supported by the simulation results in Fig. 3(b). 

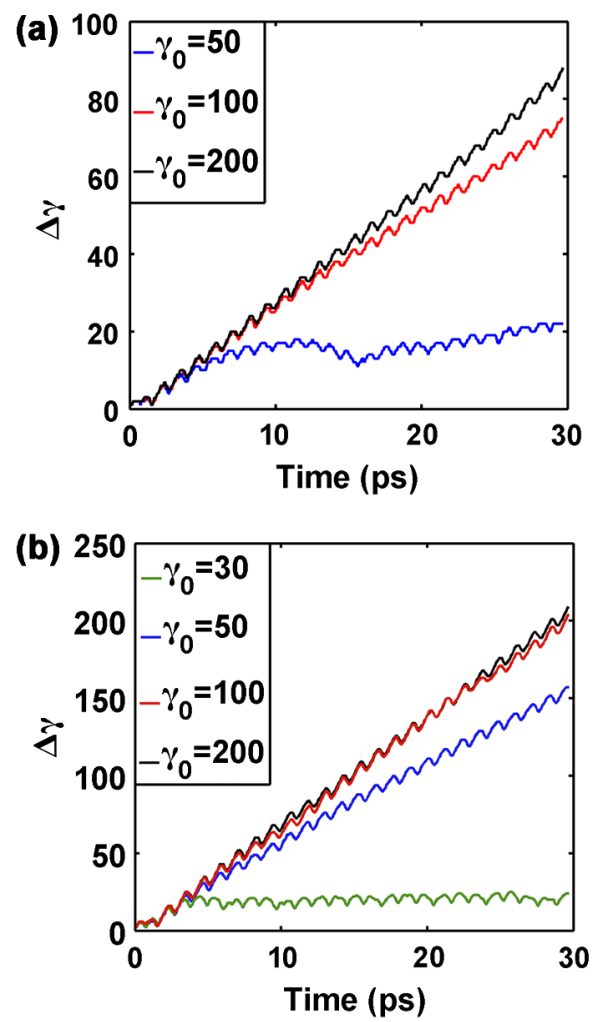

FIG. 3. Energy gain as a function of acceleration time for different initial electron energies. Here the modulation period is equal to the dephasing length. (a) $\gamma_{0}=50,100$, and 200 for $a_{0}=0.1$ (b) $\gamma_{0}=30,50,100$, and 200 for $a_{0}=0.25$.

To examine the dynamics of the entire beam, we consider phase space densities after $9 \mathrm{~mm}$ of interaction for an initial beam density. Figures 4(a) and 4(b) depict the beam distribution as a function of final longitudinal momentum and transverse position. In Fig. 4(a) the laser pulse amplitude is $a_{0}=0.25$ and the initial electron energy $\gamma_{0}=100$, while in Fig. 4(b), $a_{0}=0.1$ and $\gamma_{0}=200$. For both cases, the initial electron energy is above the threshold energy, $\gamma_{\text {th }}$. Figures 4(a) and 4(b) show that the beam electrons gaining the most energy remain transversely collimated within $5 \mu \mathrm{m}$ of the center. However, electrons that lose or maintain energy scatter significantly for $a_{0}=0.25$. Because electrons are also quasi-phase-matched to the transverse fields of the laser pulse, while there is no radial force on axis, electrons slightly off axis can be focused or defocused depending on their phase. Electrons starting in a defocusing phase will be pushed outward by the phasematched component of the transverse electric field and will not experience significant acceleration. Furthermore, for radii smaller than the spot size, the transverse force increases with radius: as electrons in the defocusing phase move off axis they experience an even larger defocusing force. Of the electrons that start in a focusing phase about half will experience acceleration and half will experience deceleration. This is seen in Figs. 4(a) and 4(b) as electrons that remain collimated on axis but have energies less than
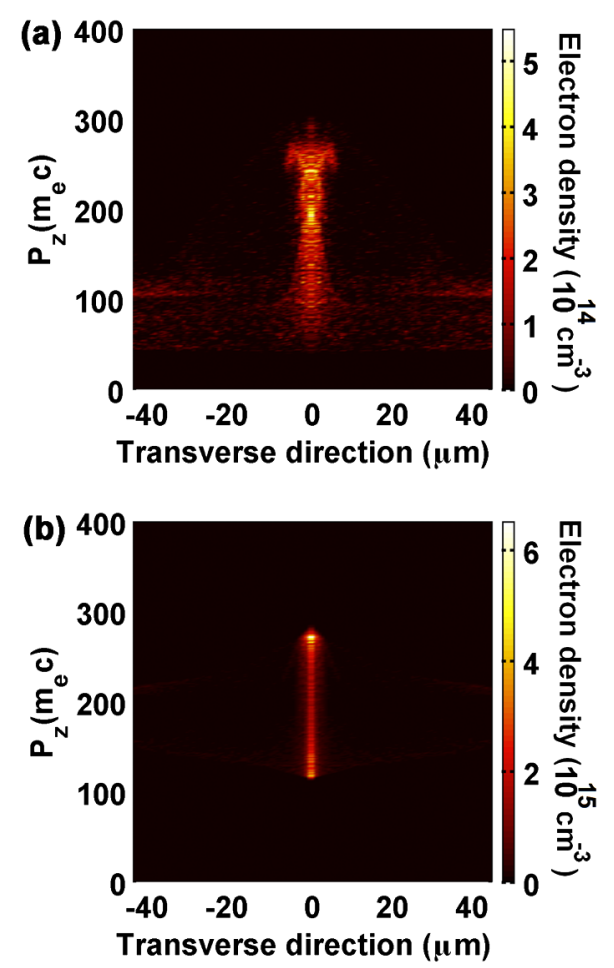

FIG. 4. Transverse position-longitudinal momentum phase space of the beam electrons after $9 \mathrm{~mm}$ acceleration for a beam density $n_{b}=3.5 \times 10^{16} \mathrm{~cm}^{-3}$, (a) $a_{0}=0.25$ and $\gamma_{0}=$ 100, and (b) $a_{0}=0.1$ and $\gamma_{0}=200$.

$\gamma_{0}=100$ and $\gamma_{0}=200$, respectively. As electrons decelerate they can drop below the threshold energy and begin to phase slip into a defocusing phase with respect to the $n=1$ spatial harmonic resulting in a scattering of lower energy electrons as seen in Fig. 4(a).

In addition to the transverse quasi-phase-matched force, there are transverse forces on the beam from the space charge of the beam, the ponderomotive force of the laser pulse on the beam, and the electrostatic potential generated by modification of the background electron density due to the laser pulse and beam. The first two we can rule out: the charge of the beams used in the simulations is $5 \mathrm{pC}$, less than space charge limit calculated previously, $40 \mathrm{pC}$ per bunch [10]. Furthermore, the ponderomotive force on the beam scales as $F_{\mathrm{pm}} \propto a_{0}^{2} / \gamma$, which because of the inverse proportionality to $\gamma$ is quite small. We then conclude that the increase in transverse scattering for $a_{0}=0.25$ as opposed to $a_{0}=0.1$ is the combination of two effects: the quasi-phase-matched transverse force scales linearly with the pulse amplitude, and the electrostatic forces due to modifications in background plasma scale quadratically with pulse amplitude. The second effect was neglected in previous works $[8,9]$.

The density perturbation in the background plasma is governed by the combined linearized continuity and momentum equations averaged over the carrier period of the laser pulse: 


$$
\frac{\partial^{2} n_{1}}{\partial t^{2}}+\omega_{p, 0}^{2} n_{1}=\frac{1}{4} n_{0} c^{2} \nabla^{2}\left|a_{0}\right|^{2}-\omega_{p, 0}^{2} n_{b}
$$

where $n_{0}$ is the averaged on-axis channel density, $\omega_{p, 0}$ is the plasma frequency, $a_{0}$ is the normalized laser vector potential, and $n_{b}$ is the beam density. For very low beam density, using the steady state approximation, and considering a radially polarized pulse the on-axis density perturbation is simply $n_{1} \simeq \frac{1}{16 \pi}\left(r_{e} w_{\mathrm{ch}}^{2}\right)^{-1} a_{0}^{2}$, where $r_{e}$ is the classical electron radius. For $a_{0}=0.1$ and $a_{0}=$ 0.25 with $w_{\text {ch }}=15 \mu \mathrm{m}, n_{1} \simeq 3.2 \times 10^{14} \mathrm{~cm}^{-3}$ and $n_{1} \simeq$ $2 \times 10^{15} \mathrm{~cm}^{-3}$, respectively. While this seems small, it actually creates a significant electrostatic force that pushes the beam electrons off axis. Using $\nabla^{2} \phi_{1}=$ $4 \pi e n_{1}$ and $\mathbf{F}=e \nabla \phi$, the electrostatic force experienced by the beam electrons is simply $\mathbf{F}_{\mathrm{es}}=\frac{1}{4} m_{e} c^{2} \nabla\left|a_{0}\right|^{2}$. The ponderomotive force of the radially polarized pulse pushes the background electrons towards the center of the channel which deflects off axis beam electrons further off axis. The electrostatic force acting alone creates an unstable equilibrium for small radii, for which a small radial electron velocity will have an early time exponential growth rate of $\left(2 \gamma_{0}\right)^{-1 / 2} a_{0} w_{\text {ch }}^{-1}$. For $a_{0}=0.25$ and $\gamma_{0}=$ 100 , one e-folding occurs after $0.8 \mathrm{~mm}$ while for $a_{0}=0.1$ and $\gamma_{0}=200$ the e-folding distance is $3 \mathrm{~mm}$.
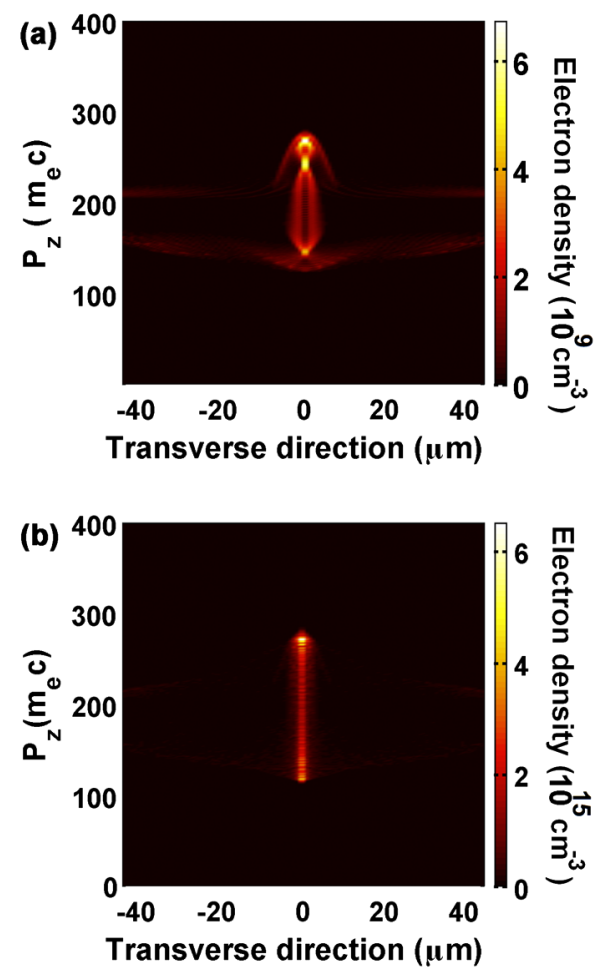

FIG. 5. Transverse position-longitudinal momentum phase space of the beam electrons after $9 \mathrm{~mm}$ acceleration for $a_{0}=$ 0.1 and $\gamma_{0}=200$ (a) low beam density, $n_{b}=7 \times 10^{10} \mathrm{~cm}^{-3}$, and (b) high beam density, $n_{b}=3.5 \times 10^{16} \mathrm{~cm}^{-3}$.
The deflection of the beam electrons can be abated by considering the charge of the electron beam. The electron beam pushes the background electrons off axis acting to cancel the ponderomotive force of the laser pulse. From Eq. (11) we see that the beam density required to balance the ponderomotive pressure of the laser is $n_{b} \simeq \frac{1}{8 \pi} \times$ $\left(r_{e} w_{\mathrm{ch}}^{2}\right)^{-1} a_{0}^{2}$. For $w_{\mathrm{ch}}=15 \mu \mathrm{m}$ and $a_{0}=0.1$, this predicts a balancing density of $n_{b} \simeq 6.3 \times 10^{14} \mathrm{~cm}^{-3}$. Figures 5 (a) and $5(\mathrm{~b})$ are a comparison of the transverse position, longitudinal momentum phase space densities after $9 \mathrm{~mm}$ of interaction for beam densities of $n_{b}=7 \times 10^{10} \mathrm{~cm}^{-3}$ and $n_{b}=3.5 \times 10^{16} \mathrm{~cm}^{-3}$, respectively. The maximum energy gain for both situations is the same, but the higher density beam has better collimation and a more monoenergetic peak.

Figure 6(a) shows the total on-axis charge density after $4.2 \mathrm{ps}$ of interaction. The red line is for $n_{b}=$ $7 \times 10^{10} \mathrm{~cm}^{-3}$ and the blue line for $n_{b}=3.5 \times 10^{16} \mathrm{~cm}^{-3}$. The higher density beam drives a plasma wave in the background plasma whose associated electric field helps to collimate the beam. The rapid oscillations in the charge density are due to microbunching of the beam electrons at the laser wavelength. This is a direct result of the focusing
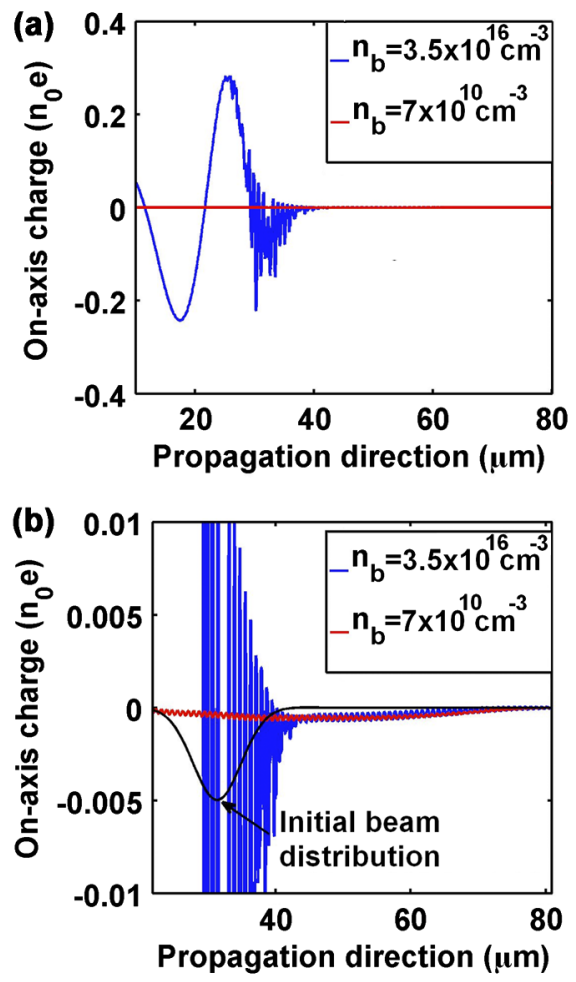

FIG. 6. On-axis charge density after 4.2 ps of acceleration with $a_{0}=0.1$. (b) Vertically magnified image of (a). The blue line is the on-axis charge density for high beam density, $n_{b}=3.5 \times 10^{16} \mathrm{~cm}^{-3}$, and the red line is for low beam density, $n_{b}=7 \times 10^{10} \mathrm{~cm}^{-3}$. The black line in (b) indicates the initial beam electron profile. 
and defocusing phases of the quasi-phase-matched transverse field. Figure 6(b) is a zoomed-in version of (a) and demonstrates the negative charge buildup on axis due to the ponderomotive force of the laser pulse. For the low density beam there is a negative charge buildup overlapping the beam, while for the high density beam there are alternating regions of positive and negative background charge, which on average reduce the deflection of the beam. The electron beam drives out the background electrons, so that positive charge remains. From Fig. 5 we see that the accelerating field was not affected by the generation of the plasma wake. We note that another way to avoid the deflection due to modifications in the background density is to lower the field intensity and propagate over a longer distance. However, lowering the field intensity increases the threshold energy for trapping which may not be ideal.

Figure 7 shows the final energy spectrum of the electron beam within the initial radius of the beam for three different initial energies: $\gamma_{0}=100, \gamma_{0}=200$, and $\gamma_{0}=500$, accelerated by a laser pulse with $a_{0}=0.1$. The beam electrons are initialized monoenergetically, and the QPMA process maintains a narrow energy spread during acceleration. The relative energy spreads, which we define as $\Delta E / E$ where $\Delta E$ is the full width half maximum, for gamma 100, 200, and 500 are $5 \%, 4 \%$, and $2.8 \%$, respectively. Since the phase velocity of the $1 \mathrm{st}$ spatial harmonic is set to $c$, the electrons with higher initial energy will remain in phase with the accelerating field over a longer duration. For example, electrons with a constant energy $\gamma=100$ will be $450 \mathrm{~nm}$ $\left(\sim \lambda_{L} / 2\right)$ delayed from an object that moves at $c$ after $9 \mathrm{~mm}$ propagation. The dephasing results in more energy spread for the lower initial energy beams.

The green curve in Fig. 7 displays the spectrum when the acceleration occurs over a longer distance: $18 \mathrm{~mm}$ for $\gamma_{0}=200$. The energy spread of the peak has dropped from $4 \%$ at $9 \mathrm{~mm}$ to $1.5 \%$ at $18 \mathrm{~mm}$, and the energy of the peak has increased. However, the monoenergetic peak no longer occurs at the maximum in the spectrum. Figure 8

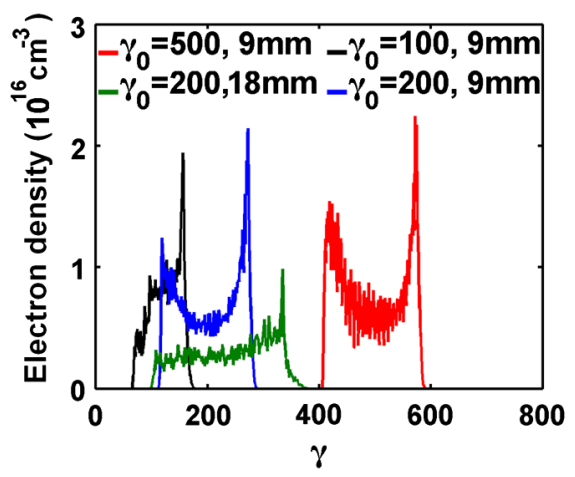

FIG. 7. Energy spectra of beam electrons for different initial energies: $\gamma_{0}=100,200$, and 500 after $9 \mathrm{~mm}$ of acceleration, are the red line, black line, and blue line, respectively, while the green line is $\gamma_{0}=200$ after $18 \mathrm{~mm}$ acceleration. In all cases the normalized vector potential was $a_{0}=0.1$.

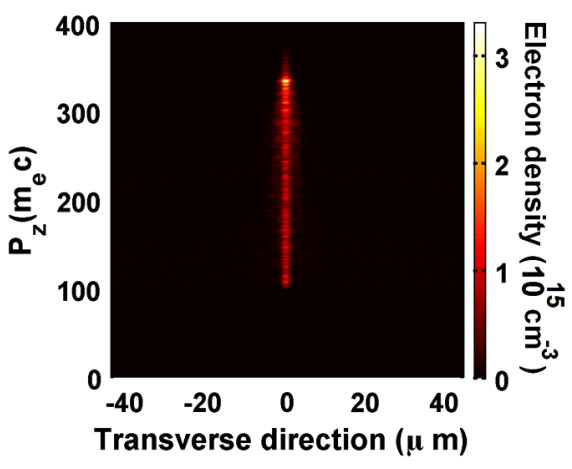

FIG. 8. Transverse position-longitudinal momentum phase space of the beam electrons after $18 \mathrm{~mm}$ of acceleration for a beam density, $n_{b}=3.5 \times 10^{16} \mathrm{~cm}^{-3}$, normalized vector potential $a_{0}=0.1$ and initial energy $\gamma_{0}=200$.

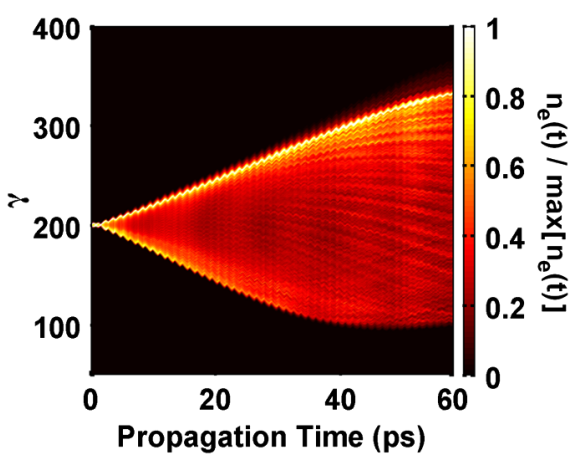

FIG. 9. Energy spectrum as a function of time for $n_{b}=3.5 \times$ $10^{16} \mathrm{~cm}^{-3}, a_{0}=0.1$, and $\gamma_{0}=200$. For clarity the spectrum is normalized to its maximum at each time.

shows the transverse position, longitudinal momentum phase space density after $18 \mathrm{~mm}$. The beam electrons remain collimated in the transverse direction and the monoenergetic peak in Fig. 7 appears as the bright dot near $330 \mathrm{MeV}$. The time evolution of the energy spectrum appears in Fig. 9. For clarity the spectrum has been normalized by the peak value at each time step. The orange line with narrow width is the monoenergetic peak. It starts as a very narrow peak and slowly broadens until $25 \mathrm{ps,} \mathrm{and}$ becomes narrow again afterward.

\section{LOW ENERGY ELECTRON ACCELERATION}

The electron beams simulated thus far have been initialized with relativistic speeds to ensure that the electrons remain phase-matched to the $n=1$ spatial harmonic. This is not favorable for small scale, tabletop laser accelerators because it requires a preliminary accelerator for injection. Thus, a method for injecting low energy electrons is critical for the realization of QPMA as a small-scale accelerator. The minimum energy of electrons in QPMA can be calculated via Eq. (10). To trap low energy electrons from the beginning and accelerate them over the entire acceleration distance requires field amplitudes for which the interaction 
between the laser pulse and background plasma becomes nonlinear, potentially destroying the slow wave structure. For example, to trap and accelerate electrons with $\gamma_{0}=20$ requires a laser pulse with a relativistic amplitude, $a_{0}=$ 0.92. Alternatively, quasi-phase-matching to low energy electrons can be achieved by gradually ramping up the phase velocity of the phase-matched spatial harmonic over the interaction length. Increasing the phase velocity can be done in several ways: increasing the electron density, increasing the modulation period, or decreasing the channel width. Here we investigate ramping the electron density.

We begin by analytically calculating the density ramp required for a particular initial electron energy. We write the density profile in terms of the plasma frequency as follows:

$$
\omega_{p}^{2}(r, z)=\omega_{p 0}^{2}+\omega_{p 1}^{2}(z)+\Gamma \omega_{p 0}^{2} \sin \left(k_{m} z\right)+\frac{4 c^{2} r^{2}}{w_{\mathrm{ch}}^{4}},
$$

where the density ramp is included in the function $\omega_{p 1}^{2}(z)$. The total wave vector for the first spatial harmonic is then $\hat{k}=k_{0}+k_{m}+\delta k-\frac{1}{2} k_{0}^{-1} k_{p 1}^{2}(z)$. The goal now is to find an expression for $k_{p, 1}^{2}(z)$ such that the energy gain is linear over the interaction length. Using $\Delta \gamma=$ $-q / m_{e} c^{3} \int \mathbf{v} \cdot \partial_{t} \mathbf{A} d t$ the energy gain is

$$
\frac{d \gamma}{d z}=k_{0} \hat{a}_{0} e^{i \int \hat{k} d z-i \omega_{0} t},
$$

where we have defined $\hat{a}_{0} \equiv 4\left(k_{0} w_{\mathrm{ch}}\right)^{-1} J_{1}(\psi) a_{0}$. For linear energy gain we require $\int \hat{k} d z-\omega_{0} t=0$, which upon differentiation with respect to $z$ provides $\hat{k}=$ $\omega_{0} / \mathrm{v}(z)$. Furthermore, we can integrate Eq. (13) to find $\gamma=\gamma_{0}+\hat{a}_{0} k_{0} z$, which allows us to find $\mathrm{v}(z)$. Putting everything together we find the ideal density ramp for a given initial energy is given by

$$
k_{p 1}^{2}(z)=2 k_{0}\left(k_{0}+k_{m}+\delta k\right)-2\left[\frac{\left(\gamma_{0}+\hat{a}_{0} k_{0} z\right)^{2}}{\left(\gamma_{0}+\hat{a}_{0} k_{0} z\right)^{2}-1}\right]^{1 / 2} k_{0}^{2},
$$

and the total wave vector for the $n=1$ spatial harmonic is simply

$$
k=\left[\frac{\left(\gamma_{0}+\hat{a}_{0} k_{0} z\right)^{2}}{\left(\gamma_{0}+\hat{a}_{0} k_{0} z\right)^{2}-1}\right]^{1 / 2} k_{0} .
$$

Care must still be taken to ensure that the total density is not less than zero, for which a sufficient condition is $(1-\Gamma) k_{p 0}^{2}+k_{p 1}^{2}\left(z=\frac{3 \pi}{2}\right)>0$.

Based on the analytical solution for the density ramp, we performed 2D cylindrical PIC simulations to investigate the trapping of low energy electrons by the laser pulse. The simulations were conducted for electrons with initial energies of $\gamma_{0}=20$ and $\gamma_{0}=50$, both of which are below the critical energy for $a_{0}=0.1$. Figure 10(a) shows the on-axis channel density for acceleration of electrons with $\gamma_{0}=20$. The value of $n_{0}$ is $2.3 \times 10^{19} \mathrm{~cm}^{-3}$ an
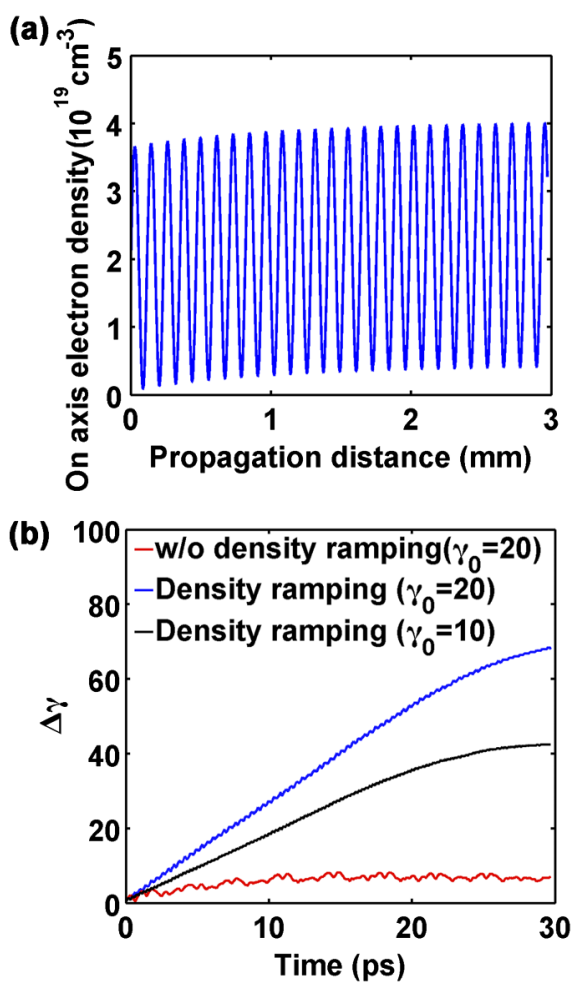

FIG. 10. (a) On-axis electron density for a plasma waveguide with a density ramp. The ramp allows trapping of lower energy electrons in this case $\gamma_{0}=20$ for a normalized vector potential of $a_{0}=0.1$. (b) Energy gain of $\gamma_{0}=20$ electrons with (blue) and without (red) the density ramp pictured in (a). The energy gain of $\gamma_{0}=10$ electrons is the black line in (b).

increase over the value used in the previous simulations, $7 \times 10^{18} \mathrm{~cm}^{-3}$. Accordingly, the modulation period is reduced to $117 \mu \mathrm{m}$ to meet the positivity condition on the density. Note the gradual increase of the averaged density over the propagation distance. In Fig. 10(b) a comparison of the energy gain of electrons with $\gamma_{0}=20$ with (blue) and without (red) the density ramp is displayed. As before, the energy gains displayed are those of electrons having the largest energy gain of all the electrons. The figure demonstrates a clear improvement of trapping electrons with below threshold energy in the presence of a density ramp. The energy gain slows down after 22 ps of interaction, because the electrons have outrun the laser pulse: the increase in average channel density decreased the group velocity of the laser pulse. The energy gain of electrons with $\gamma_{0}=10$ (black) is also displayed in Fig. 10(b). For electrons with $\gamma_{0}=10$, the same parameters for the laser and the channel were used except the laser pulse duration was increased to $600 \mathrm{fs}$, since higher density ramp is required for $\gamma_{0}=10$ than $\gamma_{0}=20$, which makes the group velocity of the laser pulse slower. While more simulations are needed to examine the robustness of the density ramp, these results show that in principle structuring the plasma density can aid in the acceleration of low energy electrons. 


\section{SUMMARY AND CONCLUSIONS}

In summary, this work examines QPMA in a corrugated plasma waveguide with fully self-consistent $2 \mathrm{D}$, cylindrical PIC simulations. The PIC code TURBOWAVE was validated by verifying the existence of spatial harmonics created by density modulations in the plasma channel. Fourier transforming the laser field yielded axial harmonics whose amplitude ratios matched analytic calculations. The local phase of the field was extracted and found to follow the density modulations as expected.

The simulations demonstrate linear acceleration of electrons by the phase-matched longitudinal electric field. The simulated acceleration gradients were $\sim 110 \mathrm{MeV} / \mathrm{cm}$ for $a_{0}=0.25$ and $\sim 50 \mathrm{MeV} / \mathrm{cm}$ for $a_{0}=0.1$, matching well with analytic predictions. Simulations also corroborated that a threshold energy exists for the trapping and linear energy gain of electrons in a spatial harmonic. Energy spectra of accelerated electron exhibited monoenergetic peaks $(\Delta E / E \approx 3 \%-5 \%)$ depending on the initial energy of the electron beam and acceleration distance. $\Delta E / E$ remains more less constant over $1.8 \mathrm{~cm}$ interaction.

The simulations revealed that the ponderomotive force due to the laser field plays a role in deflecting electrons in the accelerating phase, a result neglected in previous work [7]. The ponderomotive force of the transverse field pushes plasma channel electrons towards the axis. The resulting transverse electrostatic field defocuses the electron beam. This effect becomes more deleterious at higher pump intensity due to the increase in ponderomotive force. The defocusing was successfully mitigated by increasing the charge of the electron beam. With higher beam charge, the electron beam pushes the plasma channel electrons off axis counteracting the laser ponderomotive force.

We extended our basic model of QPMA for highly relativistic electrons to the phase-matching of lower energy electrons. An electron density ramp was used to slowly increase the phase velocity of the phase-matched spatial harmonic over the interaction length. An analytic expression for an ideal density ramp was determined and implemented in the PIC simulations. Simulations with the density-ramped modulated waveguides showed trapping of electrons with $\gamma_{0}=20$ at a pump intensity of $a_{0}=$ 0.1 . No electron energy gain was seen without the density ramp. Alternative methods for accelerating low energy electrons include increasing the modulation period or narrowing the channel over the interaction length. These will be topics of future study.

\section{ACKNOWLEDGMENTS}

This work was supported by the U.S. Defense Threat Reduction Agency, the Department of Energy, the National
Science Foundation, and the Office of Naval Research. The authors thank B. Layer for fruitful discussion.

[1] M. M. Fejer, G. A. Magel, D. H. Jundt, and R. L. Byer, IEEE J. Quantum Electron. 28, 2631 (1992).

[2] P. Serafim, P. Sprangle, and B. Hafizi, IEEE Trans. Plasma Sci. 28, 1155 (2000).

[3] H. M. Milchberg, T.R. Clark, C. G. Durfee III, T. M. Antonsen, Jr., and P. Mora, Phys. Plasmas 3, 2149 (1996).

[4] H. M. Milchberg, C. G. Durfee, III, and T. J. McIlrath, Phys. Rev. Lett. 75, 2494 (1995).

[5] J. D. Lawson, IEEE Trans. Nucl. Sci. 26, 4217 (1979); P. M. Woodward, J. Inst. Electr. Eng. 93, 1554 (1947).

[6] B. D. Layer, A. York, T. M. Antonsen, S. Varma, Y.-H. Chen, and H. M. Milchberg, Phys. Rev. Lett. 99, 035001 (2007).

[7] B. D. Layer, A. G. York, S. Varma, Y.-H. Chen, and H. M. Milchberg, Opt. Express 17, 4263 (2009).

[8] J. P. Palastro, T. M. Antonsen, S. Morshed, A. G. York, and H. M. Milchberg, Phys. Rev. E 77, 036405 (2008).

[9] J. P. Palastro and T. M. Antonsen, Phys. Rev. E 80, 016409 (2009).

[10] A. G. York, H. M. Milchberg, J.P. Palastro, and T. M. Antonsen, Phys. Rev. Lett. 100, 195001 (2008).

[11] T. Tajima and J. M. Dawson, Phys. Rev. Lett. 43, 267 (1979).

[12] S.P.D. Mangles, C.D. Murphy, Z. Najmudin, A. G. R. Thomas, J. L. Collier, A.E. Dangor, E. J. Divall, P. S. Foster, J. G. Gallacher, C. J. Hooker, D. A. Jaroszynski, A. J. Langley, W. B. Mori, P. A. Norreys, F. S. Tsung, R. Viskup, B. R. Waltonand and K. Krushelnick, Nature (London) 431, 535 (2004).

[13] C. G. R. Geddes, Cs. Toth, J. van Tilborg, E. Esarey, C. B. Schroeder, D. Bruhwiler, C. Nieter, J. Cary, and W.P. Leemans, Nature (London) 431, 538 (2004).

[14] J. Faure, Y. Glinec, A. Pukhov, S. Kiselev, S. Gordienko, E. Lefebvre, J.-P. Rousseau, F. Burgy, and V. Malka, Nature (London) 431, 541 (2004).

[15] E. Esarey, C. B. Schroeder, and W. P. Leemans, Rev. Mod. Phys. 81, 1229 (2009).

[16] W.P. Leemans, B. Nagler, A. J. Gonsalves, Cs. Toth, K. Nakamura, C. G. R. Geddes, E. Esarey, C. B. Schroeder, and S. M. Hooker, Nature Phys. 2, 696 (2006).

[17] J. Faure, C. Rechatin, A. Norlin, A. Lifschitz, Y. Glinec, and V. Malka, Nature (London) 444, 737 (2006).

[18] M. Chen, Z.-M. Sheng, Y.-Y. Ma, and J. Zhang, J. Appl. Phys. 99, 056109 (2006).

[19] A. Pak, K. A. Marsh, S. F. Martins, W. Lu, W. B. Mori, and C. Joshi, Phys. Rev. Lett. 104, 025003 (2010).

[20] W. D. Kimura, G. H. Kim, R. D. Romea, L. C. Steinhauer, I. V. Pogorelsky, K. P. Kusche, R. C. Fernow, X. Wang, and Y. Liu, Phys. Rev. Lett. 74, 546 (1995).

[21] T. Plettner, R. L. Byer, E. Colby, B. Cowan, C. M. S. Sears, J.E. Spencer, and R. H. Siemann, Phys. Rev. Lett. 95, 134801 (2005).

[22] E. Esarey, P. Sprangle, and J. Krall, Phys. Rev. E 52, 5443 (1995). 
[23] B. Hafizi, A. Ting, E. Esarey, P. Sprangle, and J. Krall, Phys. Rev. E 55, 5924 (1997).

[24] P. Sprangle, E. Esarey, and J. Krall, Phys. Plasmas 3, 2183 (1996).

[25] D. F. Gordon, IEEE Trans. Plasma Sci. 35, 1486 (2007).
[26] K. S. Yee, IEEE Trans. Antennas Propag. 14, 302 (1966).

[27] C. K. Birdsall and A. B. Langdon, Plasma Physics via Computer Simulation (McGraw-Hill, New York, 1985). 